\title{
LA PRODUCCIÓN DE TEXTOS ARGUMENTATIVOS Y SU VÍNCULO MOTIVACIONAL CON EL APRENDIZAJE
}

\author{
The Production of Argumentative texts and its Motivational link with Learning
}

\author{
Aleydy Esther Ramos Sánchez, MsC. \\ Universidad César Vallejo, Perú \\ https://orcid.org/0000-0001-6834-9741 \\ p2000068923@ucvvirtual.edu.pe
}

\author{
Gilberto Carrión-Barco, MsC. \\ Universidad César Vallejo, Perú \\ https://orcid.org/0000-0002-1104-6229 \\ ccarrionba@ucvvirtual.edu.pe
} Palabras claves: Producción de Textos Argumentativos, Estrategias Motivacionales, Educación Recibido: 30 de diciembre de 2020
Secundaria.

Keywords: Production of Argumentative Texts, Motivational Strategies, Secondary Education.
Aceptado: 18 de marzo de 2021

\section{RESUMEN}

Las instituciones educativas afrontan la necesidad de incorporar nuevas prácticas pedagógicas que favorezcan una adecuada producción de textos en los estudiantes, frente a esta realidad se requiere potenciar los esfuerzos educativos reconociendo la importancia que tienen para alcanzar el desarrollo del enfoque comunicativo, más aún en el contexto actual donde la educación muestra desigualdades en la adquisición de los aprendizajes. Frente a ello, el compromiso de los docentes de incluir técnicas elementales y estrategias motivacionales que permitan la producción de textos argumentativos, donde los estudiantes sean capaces de replicar críticamente sobre cualquier tema de interés con coherencia y propiedad. La investigación tuvo como objetivo Diseñar una estrategia motivacional significativa para mejorar la producción de textos argumentativos de los estudiantes del segundo grado de secundaria. Para lograr el objetivo propuesto se desarrolló una metodología de tipo aplicada, con alcance descriptivo, basada en el enfoque cuantitativo con un diseño de tipo no experimental transaccional. Como instrumento, un cuestionario con 30 ítems organizado en tres dimensiones y como técnica de recolección de la información, la encuesta; realizado el proceso de análisis documental. Los resultados obtenidos nos llevan a identificar que el $59 \%$ de los estudiantes se encuentran en el nivel inicio y el $9 \%$ en satisfactorio. Ante esto, se llega a la conclusión que es necesario trabajar estrategias motivacionales hacia el aprendizaje, como realización de la tarea y como entretención para contribuir a la mejora de la producción de textos argumentativos.

\begin{abstract}
Educational institutions face the need to incorporate new pedagogical practices that favor an adequate production of texts in students, in the face of this reality it is necessary to strengthen educational efforts, recognizing the importance they have to achieve the development of the communicative approach, even more so in the context current where education shows inequalities in the acquisition of learning. Faced with this, the commitment of teachers to include elementary techniques and motivational strategies that allow the production of argumentative texts, where students are able to critically replicate on any topic of interest with coherence and property. The objective of the research was to Design a meaningful motivational strategy to improve the production of argumentative texts of second-grade students. To achieve the proposed objective, an applied-type methodology was developed, with a descriptive scope, based on the quantitative approach with a non-experimental transactional design. As an instrument, a questionnaire with 30 items organized in three dimensions and as an information gathering technique, the survey; carried out the document analysis process. The results obtained lead us to identify that $59 \%$ of the students are at the beginning level and $9 \%$ are satisfactory. Given this, the conclusion is reached that it is necessary to work motivational strategies towards learning, as a task performance and as entertainment to contribute to the improvement of the production of argumentative texts.
\end{abstract}




\section{INTRODUCCIÓN}

La calidad educativa y la sociedad del conocimiento son dos conceptos que están muy asociados y tienen una complementariedad recíproca (Gutiérrez y Uceda, 2017). La producción de textos escritos es un reto fundamental por el cual debe un estudiante atravesar y constituye en gran parte un desempeño exitoso del individuo en su vida tanto académica como profesional, a pesar de los esfuerzos que se han realizado para mejorar esta capacidad en los escolares de la educación básica, muchos investigadores muestran gran preocupación por el rendimiento deficiente que muestran tanto los jóvenes en esta materia (Villasmil et al., 2009).

Esta problemática se evidencia en varios de los países de América Latina, por ejemplo, Chile, la producción de textos argumentativos en los estudiantes evidencia escasa justificación del conocimiento proposicional transmitido en el texto y justificación mediante argumentos únicamente del tipo razonamiento y aplicación de reglas a casos, lo que muestra la problemática en la producción de textos argumentativos (Araya \& Orellana, 2019).

En el caso de Brasil, las estrategias usadas por parte de los maestros no se evidencian hasta el décimo año de estudios, la investigación ha demostrado que esta línea de argumentación es falaz, una de las recomendaciones es enfatizar en la importancia de los ajustes curriculares para incluir la enseñanza de la argumentación desde los primeros años de escolaridad (Riolfi \& Costa, 2018).

En cuanto a lo que sucede en Colombia, la situación es preocupante para los estudiantes, ya que no tienen conocimiento sobre la superestructura de los diferentes tipos de textos, carecen de argumentos en su redacción (Pulgarín, 2018).

En los hallazgos de las evaluaciones nacionales e internacionales y los resultados de investigaciones relacionadas al logro de aprendizaje de los estudiantes, se evidencia el bajo rendimiento de los alumnos del nivel primaria y secundaria en comprensión lectora.

Así se manifiesta, en el documento de la evaluación PISA difundido por la Unidad de Medición de la Calidad educativa (2018), señala que un gran porcentaje de estudiantes menores de 15 años no están logrando el nivel esperado en la competencia lectora. Así, los porcentajes de alumnos que se ubican en los niveles 1a, $1 \mathrm{~b}$ y debajo del $1 \mathrm{~b}$ presentan diferencia, así el $29,5 \%$ (Chile) y $72,1 \%$ (República Dominicana).

En Perú, el 54,4\% de estudiantes se encuentra en estos niveles de desempeño, los porcentajes reportados dan cuenta de las deficiencias que tienen los estudiantes respecto a la lectura, situación que pone en evidencia de la utilización de estrategias motivacionales con la intención de enriquecer la producción de textos en este grupo de estudiantes.

En la Evaluación Censal (ECE) y la Evaluación Muestral (EM) realizada por el MINEDU (2018), en este año el 2018, el $20,2 \%$ de estudiantes se ubicó dentro del logró los aprendizajes esperados para el segundo grado de secundaria en escritura, un gran porcentaje de estudiantes evaluados $(80,2 \%)$ redactó un escrito con suficiencia de información, sin digresiones, repetición innecesaria de ideas o contradicciones.

Las principales deficiencias de los estudiantes se evidencian en la utilización de conectores, referentes y signos de puntuación. Solo el $37,7 \%$ de los estudiantes usó correctamente los conectores y los referentes; y solo el 25,3 \% utilizó en forma correcta los signos de puntuación, lo que genera una producción de textos argumentativos de niveles en procesos; esto podría mejorar si se aplican estrategias motivacionales que permitan el desarrollo de las capacidades o habilidades comunicativas sumamente necesarias para que las personas logren dentro de la sociedad o espacios donde interactúan un exitoso desenvolvimiento.

En el caso de la institución educativa "Mariscal Castilla" los estudiantes de segundo grado de secundaria, presentan en argumentación una gran debilidad y esto se ve reflejado en el record de notas obtenidos en el año 2019, y sustentado por la evaluación ECE el 2019 el $47.7 \%$ se encuentra en inicio, el $27,7 \%$ en proceso y el 11,2\% satisfactorio existiendo un vínculo directo entre la comprensión lectora y la producción de textos, se pudo evidenciar que los estudiantes redactan variedad de textos escritos en todas las asignaturas y que la composición textual se concentra en la producción de textos expositivos y mapas conceptuales, siendo los textos argumentativos los que menos se realizan en los salones de clase.

En cuanto a los procedimientos que siguen al componer un texto escrito, la mayor parte solo hizo mención a la fase de escritura o redacción; con respecto a la planificación se limitaron a la recolección de datos y, sobre la revisión solo destacaron en la modificación de aspectos formales del texto. 
Otro aspecto observado en la producción de textos es la dificultad existente en cuanto a la baja calidad que tienen los textos argumentativos que producen los estudiantes de la institución educativa Mariscal Castilla, pues la mayoría de ellos al no escribir un texto de buena calidad insta de lejos a tener la capacidad que se requiere para escribir y que es fundamental para su desarrollo como emitir su punto de vista sobre su contexto y han permitido ver un desfase del escribir , es por ello que impera que lo aborde como problema y amerite sea investigado desde la acción.

Es una realidad, las dificultades con la producción de textos argumentativos que presentan los estudiantes, lo que se convierte en un obtaculo para el avance académico y personal. Es por ello que se planteó como objetivo diseñar una estrategia motivacional significativa para mejorar la Producción de Textos Argumentativos de los estudiantes del segundo grado de secundaria. Estas propuestas sobre estrategias motivacionales hacen posible la innovación, lo cual determina la imaginación y creatividad de los estudiantes y docentes, asimismo la construcción de un instrumento que podrá usarse como modelo por otros investigadores.

\section{DESARROLLO}

\section{Marco teórico}

Con respecto al aporte teórico es relevante en la presente investigación, el enfoque comunicativo, permite abordar la enseñanza de la lengua de una manera más significativa y funcional, así una de las consideraciones centrales del enfoque es que el uso de textos tiene lugar en situaciones de comunicación definidas y con propósitos claros. Por esta razón, nociones como las de emisor, destinatario, intención comunicativa, contexto verbal, situación o conocimiento del mundo empiezan a cobrar mayor importancia (Camps, 1995). Otro de los enfoques sumamente importantes de la enseñanza de la lengua se enfoca en desarrollar la competencia lingüística Noam Chomsky.

Según esta teoría, la competencia es el sistema de reglas lingüísticas, interiorizada por los hablantes, que conforma sus conocimientos verbales y que le permite entender un número infinito de enunciados lingüísticos (Pérez $\mathrm{F}$. et al., 2019). El concepto de competencia comunicativa fue propuesto por el etnógrafo (Hymes, 1972), para explicar que se necesita otro tipo de conocimientos, aparte de la gramática, para poder usar el lenguaje con propiedad.

Y es que, a la A la par de la comprensión lectora, también está la necesidad de desarrollar la producción de textos escritos (Merma, 2018). Sin duda estos dos aspectos juegan un rol importan en la producción de textos argumentativos, según la revisión de la literatura se observa distintas definiciones, la argumentación se encuentra asociada a las habilidades comunicativas y está presente en el contexto de la vida cotidiana (Weston, 2005), el texto argumentativo es una de las capacidades o competencias comunicativas de mucha importancia y necesarias para el desarrollo del individuo.

Dentro del enfoque comunicativo, el objetivo del desarrollo de las competencias de comprensión y producción de textos es la construcción de significados, lo que supone ir más allá de la concepción habitual de competencia lingüística o gramatical (Cárdenas P., 2017) refuerza los sustentos del enfoque comunicativo aduciendo que el saber con el que deberían contar los estudiantes sobre la lengua y el funcionamiento de sus elementos (teoría gramatical y aspectos normativos) no es suficiente y propone el uso del lenguaje en sus diferentes formas como horizonte de trabajo.

Otro de los enfoques importantes es la competencia argumentativa se entiende como la integración de conocimientos, habilidades y actitudes, movilizados en la defensa o rechazo de una aseveración, hecho o conclusión a través de una exposición coherente, fundamentada y sistemática con el objeto de plantear una postura a la vez que se comparten significados y se negocia un consenso (Guzmán-Cedillo et al., 2013).

Porque en el ámbito de la formación formal existen necesidades de desarrollar la competencia argumentativa utilizando las formas didácticas, para que el estudiante utilice información bajo sustentos, pruebas y evidencias.

También se puede indicar como otro de los enfoques importantes en la producción de textos argumentativos, es la Psicología Cognitiva, a pesar de que los estudiantes tienen buenos conocimientos de gramática y dominaban los diferentes usos de la lengua, sus resultados en composición no eran satisfactorios, ante esta situación, surge la necesidad por conocer los distintos procesos del pensamiento superior que se ponen en juego al momento de escribir, Flower \& Hayes, (1980) de los análisis de sus observaciones se dedujo que los escritores competentes desarrollan una gran actividad metacognitiva durante el proceso de composición, así pues: hacen una representación de la tarea teniendo en cuenta la audiencia, la función comunicativa y el contexto. 
Revisan el texto para ver si se adapta a los objetivos planteados y manifiesta lo que ellos quieren transmitir. El modelo plantea dos estrategias: de decir el conocimiento y estrategias de construir el conocimiento; estas últimas, según estos autores, son las verdaderas prácticas escritoras generadoras de aprendizaje.

Díaz Rodríguez (2006) hace hincapié en que la argumentación es una forma de convencer o lograr una adhesión de un determinado auditorio, pero con apoyo fundamentalmente en criterios racionales, en ese sentido Villasmil et al., (2009),lo agrupan dentro de las capacidades importantes de la comprensión lectora, este proceso implica incluir o integrar los componentes de un texto (lingüísticos y extralingüísticos) en una representación semántica unificada, requiere, por consiguiente, de deducciones, del establecimiento de relaciones de causa y efecto, correlaciones y comparaciones de ideas.

Por su parte Rosado (2012), explica que, un texto argumentativo es una organización textual centrada en el juicio y en la toma de una postura respecto de algún asunto polémico. La finalidad del autor puede ser probar o demostrar una idea (o tesis), refutar la contraria o bien persuadir o disuadir al receptor sobre determinados comportamientos, hechos o ideas. A decir de Román F. \& Zapata F. (2019) I a argumentación se constituye como el mecanismo discursivo que une la construcción del conocimiento en todas las áreas, constituye una ventaja en el desarrollo de temas disciplinares en la ejecución de tareas indicadas, en las que es primordial el razonamiento reflexivo y crítico.

Dentro de las propiedades del texto argumentativo, según Cassany, Luna y Sanz (2007) hacen la siguiente propuesta respecto a las propiedades, una de las primeras es la adecuación, el texto es adecuado en tanto se ha redactado con eficiencia y cumpla con los criterios básicos para los cuales fue escrito; la coherencia requiere de los elementos pragmáticos para manifestarse, así un texto será coherente dentro de un contexto en particular, fuera de este podría resultar incoherente.

Respecto a la propiedad cohesión textual: relacionada con la unión, concatenación e imbricación de los elementos internos del texto (párrafos e ideas). La cohesión hace referencia a las articulaciones gramaticales del texto (puntuación, conjunciones, conectores lingüísticos). La concisión: entendida como la propiedad o característica que otorga brevedad al texto, impide la excesiva redundancia y evita rodeos inútiles.

Respecto a los procesos de escritura, Figueroa \& Simón (2011) y Portal (2010) concuerdan en que la mayoría de estudiantes al producir un texto siguen tres procesos importantes que van entrelazados planificación del texto, textualización y revisión. El proceso de planificación corresponde al proceso mediante el cual se realizan toda la programación de las actividades a desarrollar para la elaboración del texto, esta capacidad permite estar anticipados en la forma, el contenido, el tema, el tipo de texto que se pretende desarrollar, los recursos textuales que se van a utilizar y hasta los argumentos que tendrá el texto.

Para Morales y Ramírez (2019) La capacidad de argumentación es una competencia altamente demandada que se hace necesaria en todos los procesos de selección (p.8) La producción de textos argumentativos se sustenta desde el enfoque comunicativo. El enfoque comunicativo, responde a las teorías socio constructivistas, constructivistas y teorías emergentes de las corrientes contemporáneas (Delval, 2017). Así que, la Teoría del Aprendizaje Significativo de David Ausubel, el origen de esta está en el interés que tiene Ausubel por conocer y explicar las condiciones y propiedades del aprendizaje, que se pueden relacionar con formas efectivas y eficaces de provocar de manera deliberada cambios cognitivos estables, susceptibles de dotar de significado individual y social.

Por su parte la teoría Sociocultural de Lev Semionovich Vigotsky; reconoce que el aprendizaje es un proceso personal, pero afirma que el autoconstrucción de conocimientos se logra en la interacción entre el sujeto y el contexto sociocultural en que se desenvuelve, el que luego se internaliza. Ante estos postulados surgen la estrategia motivacional basada en el enfoque motivación escolar, Robbins et al., 2004 y Hattie, 2009) señalan que esta teoría va en crecimiento en el ámbito educativo y se reconoce como un factor importante en el aprendizaje.

La motivación requiere de la disposición emocional Golleman, hace mención de la inteligencia emocional, es la forma de interactuar de las personas en razón a sus sentimientos y predispone para controlar los impulsos. En ese caso se desarrolla habilidades como la autodisciplina (Calsamiglia, 2008). La inteligencia emocional es asumida como una teoría de la motivación, dado que el escritor debe controlar sus impulsos. Difícilmente podrá producirse un texto, si el estudiante está con ira, cólera, deprimido, exaltado alegremente.

Golleman recomienda el control emocional. El docente debe asumir como estrategias de motivación, una práctica pedagógica que se centre en la retroalimentación formativa. Anijovich y Cappelletti (2017), recomienda que el docente debe ser amable, empático, proactivo con los estudiantes. Concuerda con el planteamiento del Currículo Nacional del Ministerio de Educación del Perú, cuando sostiene el uso constructivo del error. El error, debe ser una oportunidad para 
mejorar y esto se percibirá al redactar el texto, seguramente que habrá problemas de ortografía, de coherencia del texto, en ese sentido, el docente debe hacer del error como una oportunidad para escribir textos en forma adecuada.

La motivación es el producto de la interacción entre el individuo y la situación que lo rodea (Naranjo, 2009). Entonces, el producir textos, debe ser un proceso de construcción de aprendizaje, dado que se activan procesos mentales como el reconocer el tipo de texto, analizar ideas, recordar, diferenciar ideas de los diversos argumentos de los autores, sintetizar, parafrasear y así evitar copiar y pegar ideas.

La interacción entre el estudiante y el momento que está viviendo, es clave. Por otro lado, los docentes deben tomar en cuenta como estrategias para la motivación, las características de sus estudiantes, en este caso, es aceptar que, al ser estudiantes del segundo grado de secundaria, son adolescentes, por lo que plantear retos para construir como textos argumentativos, la de redactar sobre el aborto, el enamoramiento, etc. será muy atractivo para ellos (Gálvez, 2017).

El enfoque comunicativo, establece que las competencias se desarrollan en forma interdependiente, Cassany lo advierte, dado que, para la competencia escrita, se articulan la expresión oral y la comprensión lectora. La planificación curricular indica que los docentes deben asumir la oralidad y la compresión lectora como competencias básicas para la producción de textos. El lenguaje Integral es una teoría emergente para la producción de textos, Kenneth Goodman, sostiene desde la perspectiva de Emilio Ferreyro, que el enfoque comunicativo, tiene como propósito la de formar estudiantes escritores de textos. Por otra parte, para Sánchez, (1997) citado por Mejías, (2019) Afirman que la escritura es de vital importancia para el estudiante ya que enfrenta con mayor éxito su formación académica y su desempeño profesional (p. 15)

Los textos escritos y su proceso de lectura constituyen una parte movilizadora y constructiva para el aprendizaje. Los escritores que inician este proceso deciden sobre temas que le interesen a los lectores o a la audiencia que se dirigen, en este caso, deducen lo que el escritor intenta decir, así como valorar lo que encuentra en el texto. Los textos que se redactan deben ser reales, del contexto, que tengan su listado de palabras con secuencia fónica. Los escritores deben saber a qué público se dirigen, inferir su sensibilidad y los lectores se identifican con sus autores, estableciendo así una relación entre el escritor auténtico y el lector (Tabash, 2017).

\section{Metodología}

La presente investigación, se desarrolló bajo el enfoque cuantitativo que usa la recolección de datos para verificar hipótesis con base en la medición numérica y el análisis estadístico, con la finalidad de establecer teorías (Hernández et al., 2014).

El diseño fue no experimental en la que el investigador observa cómo se desarrollan los sucesos tal y como se desenvuelven en el contexto y examinarlas Calderón y Alzamora (2018), se usó el tipo de diseño transeccional descriptivo que, estudia los acontecimientos de las practicas, grados, niveles de una o más variables en una agrupación de datos para analizarlos (Rodríguez y Mendivelso, 2018).

La población estuvo conformada por 796 estudiantes de la Institución Educativa - Paita. Este registro se tomó de las listas oficiales del Sistema de información de Apoyo a la Gestión de la Institución Educativa 2020 (MINEDU, 2020).

El criterio de inclusión estuvo formado por 161 estudiantes del segundo grado de secundaria de las secciones A B C D y E. Las estudiantes que no pertenecen al segundo grado de secundaria de las secciones A B C D y E. La muestra fue conformada por 34 estudiantes de segundo grado del nivel secundaria, sección "A".

El muestreo es la base de la inferencia estadística, cuyo objetivo es proporcionar modelos matemáticos que extiendan las conclusiones de estudios realizados en una parte de la población, en este caso, muestra. Se utilizó el muestreo no probabilístico de tipo intencionada, la selección de los sujetos del estudio depende de ciertas características y criterios, que la investigadora consideró en el momento de su aplicación (Batanero, Gea y Begué, 2019). Por consiguiente, en el presente estudio se seleccionó a los estudiantes del segundo grado A de secundaria ya que se con contó con acceso a la información y la realidad de los estudiantes.

El instrumento de recopilación de datos, fue la encuesta con 30 interrogantes, con el fin de recolectar datos o información sobre la producción de textos argumentativos. Se definió la confiabilidad trabajando con el coeficiente Omega de McDonald a través del software Jamovi, 1.2.27; se trabajó con este coeficiente porqu el resultado es ordinal (Frías-Navarro 2019).

Para el trabajo de investigación el coeficiente omega de McDonald fue de 0.973 , lo que confirma que tiene alta confiabilidad. 


\section{Resultados}

A continuación, se presentarán los resultados distribuidos en tablas de frecuencias y porcentajes de la encuesta sobre Producción de textos argumentativos en los estudiantes de segundo grado de educación secundaria de la IE Mariscal Castilla.

Tabla 1. Producción de textos argumentativos

\begin{tabular}{llcc}
\hline Rango & Calificación & \multicolumn{2}{c}{ VD: Producción de textos argumentativos } \\
\hline \multirow{3}{*}{$0-47$} & Inicio & Frecuencia $(\mathrm{f} 1)$ & Porcentaje $\%$ \\
$48-75$ & Proceso & 20 & $59 \%$ \\
$76-100$ & Satisfactorio & 11 & $32 \%$ \\
& Total & 3 & $9 \%$ \\
& 34 & $100 \%$ \\
\hline
\end{tabular}

Figura 1. Producción de textos argumentativos

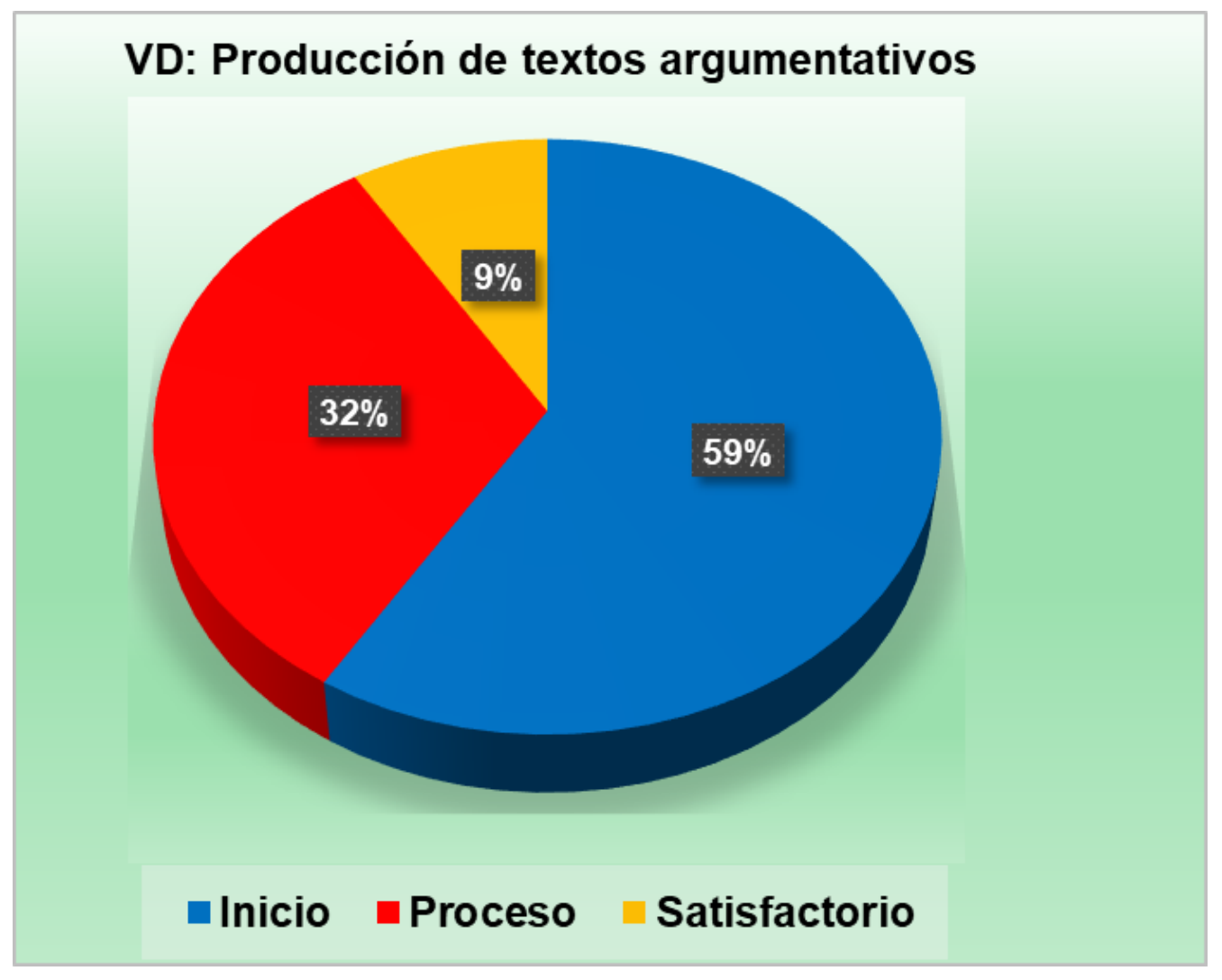

En la tabla 1 y la figura 1 se observa que del total de estudiantes encuestados del segundo grado de educación secundaria de la IE Mariscal Castilla, el 59\% se sitúa en un nivel Inicial, el 32\% se encuentran en Proceso y el $9 \%$ se evidencia en el nivel satisfactorio con respecto a la producción de textos argumentativos. 
Tabla 2. Dimensión propiedades del texto argumentativo

\begin{tabular}{llcc}
\hline Rango & Calificación & \multicolumn{2}{c}{ D1: Propiedades del texto argumentativo } \\
\hline \multirow{2}{*}{$0-47$} & Inicio & Frecuencia $(\mathrm{f1})$ & Porcentaje $\%$ \\
$48-75$ & Proceso & 26 & $76 \%$ \\
$76-100$ & Satisfactorio & 5 & $15 \%$ \\
& Total & 3 & $9 \%$ \\
& & 34 & $100 \%$ \\
\hline
\end{tabular}

Figura 2. Dimensión propiedades del texto argumentativo

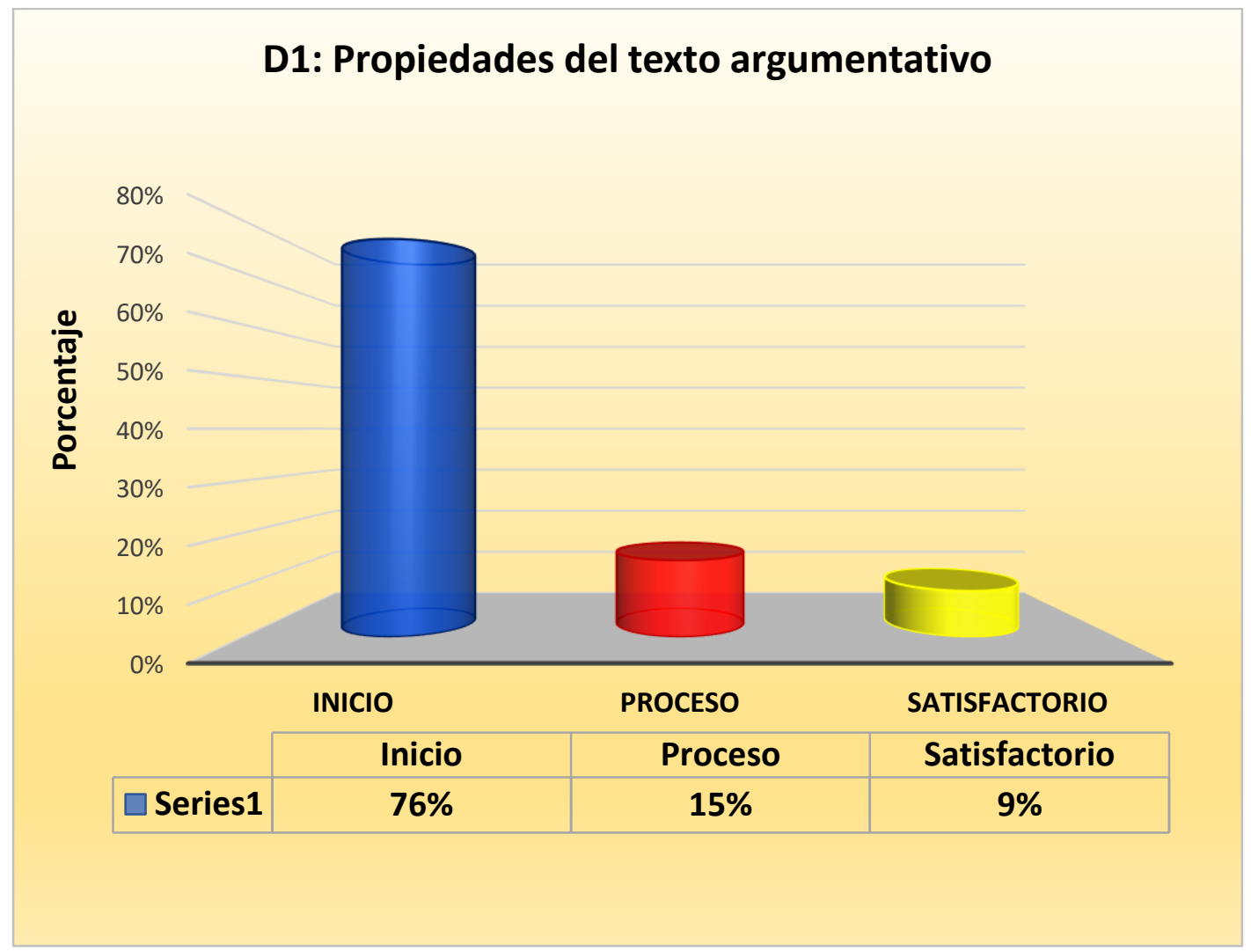

En la tabla 2 y la figura 2 se observa que del total de estudiantes encuestados del segundo grado de educación secundaria de la IE Mariscal Castilla, el $76 \%$ se sitúa en un nivel Inicial, el 15\% se encuentran en Proceso y se evidencia el nivel Satisfactorio en $9 \%$ con respecto a las propiedades del texto argumentativo. 
Tabla 3. Dimensión Procesos de escritura

\begin{tabular}{llcc}
\hline Rango & Calificación & \multicolumn{2}{c}{ D2: Procesos de escritura } \\
\hline \multirow{3}{*}{$0-47$} & Inicio & Frecuencia (f1) & Porcentaje \% \\
$48-75$ & Proceso & 22 & $65 \%$ \\
$76-100$ & Satisfactorio & 9 & $26 \%$ \\
& Total & 3 & $9 \%$ \\
& & 34 & $100 \%$ \\
\hline
\end{tabular}

Figura 3. Dimensión Procesos de escritura

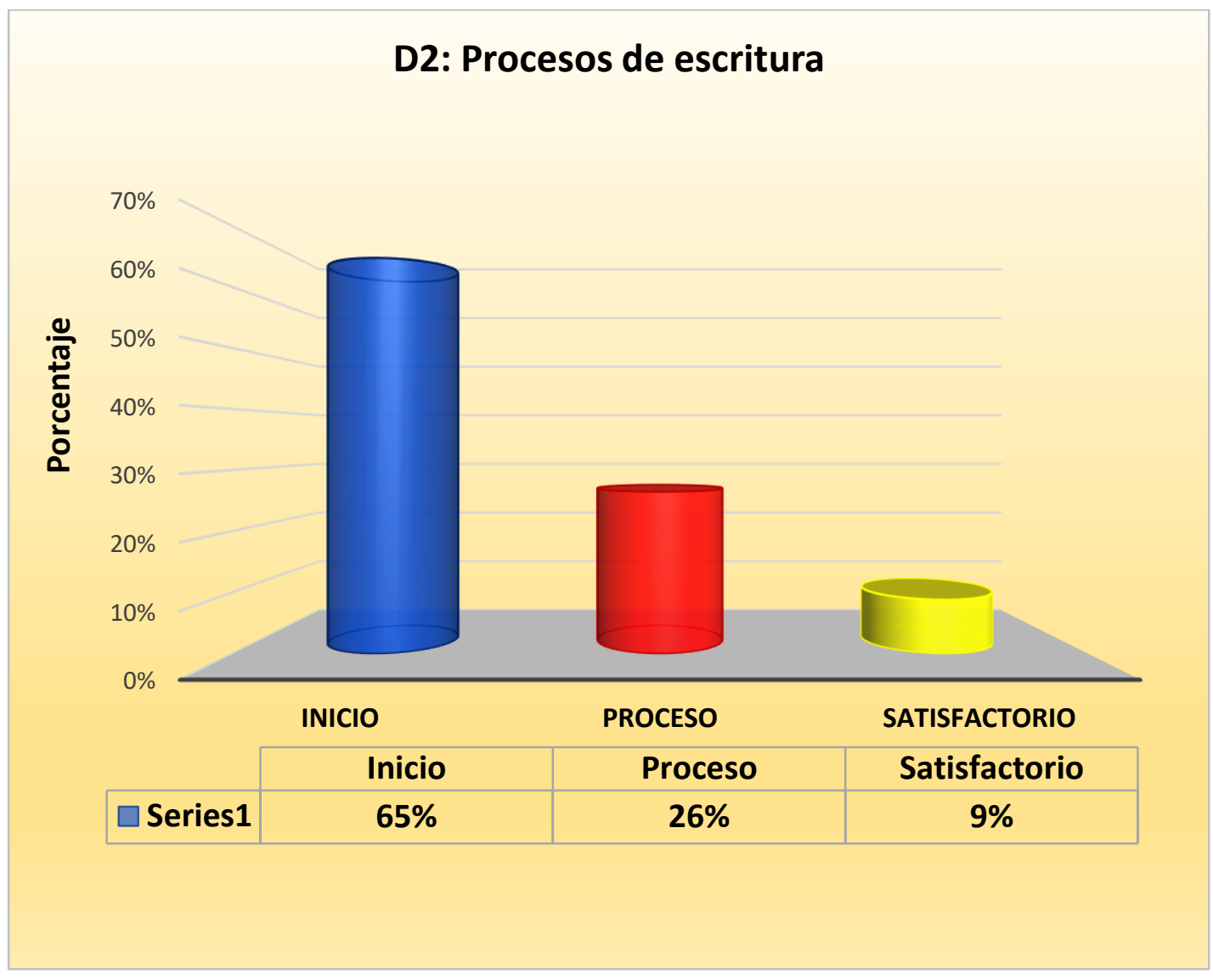

En la Tabla 3 y la Figura 3 se observa que del total de estudiantes encuestados del segundo grado de educación secundaria de la IE Mariscal Castilla, el $65 \%$ se sitúa en un nivel inicial, el $26 \%$ se encuentran en proceso y se evidencia que el $9 \%$ está en el nivel satisfactorio con respecto al proceso de escritura de textos argumentativos. 
Tabla 4. Características del texto argumentativo

\begin{tabular}{clcc}
\hline Rango & Calificación & D3: Características del texto argumentativo \\
\hline \multirow{2}{*}{$0-48$} & & Frecuencia $(\mathrm{f} 1)$ & Porcentaje \% \\
$49-75$ & Inicio & 20 & $59 \%$ \\
$76-100$ & Proceso & 11 & $32 \%$ \\
& Satisfactorio & 3 & $9 \%$ \\
& Total & 34 & $100 \%$ \\
\hline
\end{tabular}

Figura 4. Características del texto argumentativo

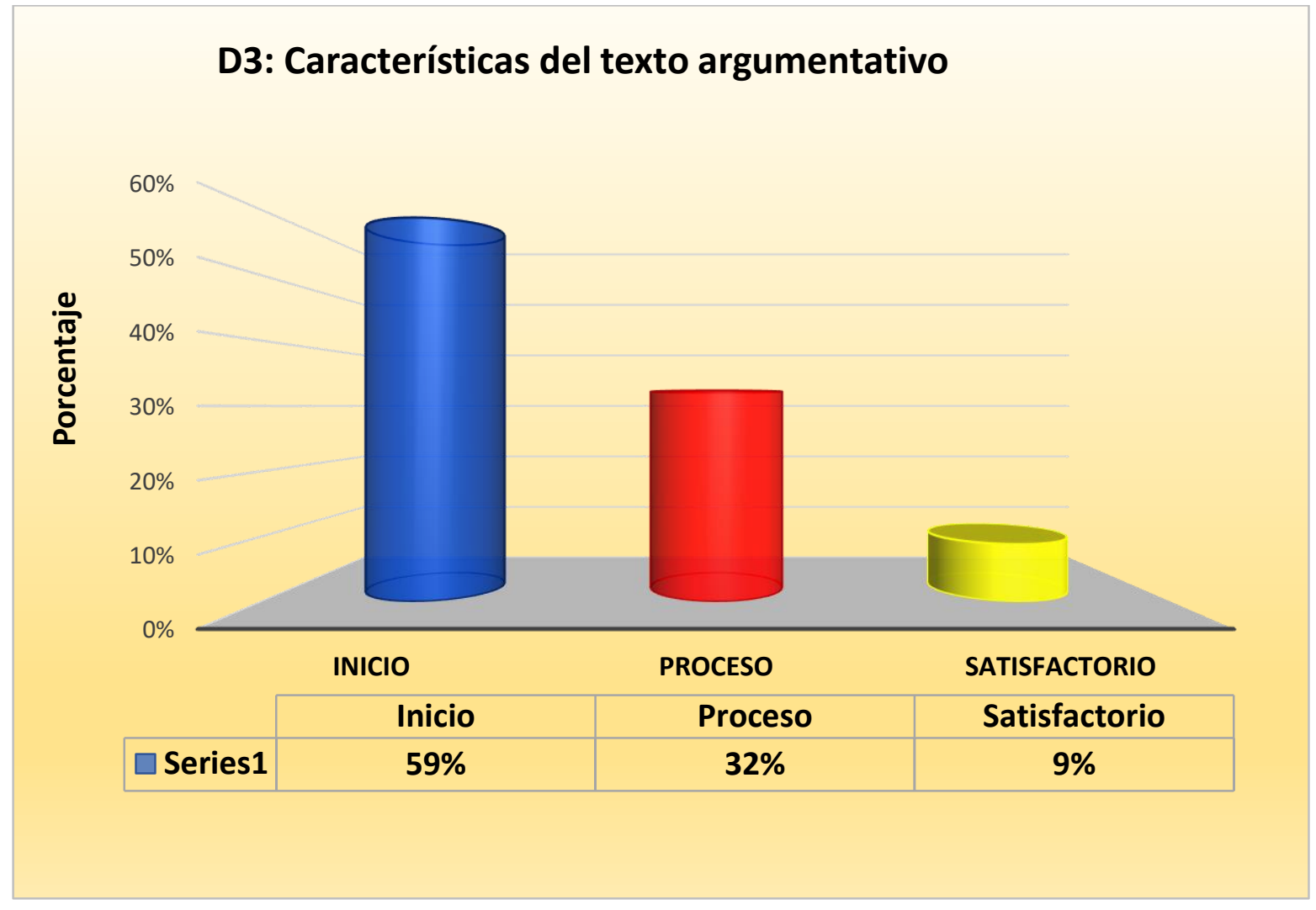

En la Tabla 4 y la Figura 4 se observa que del total de estudiantes encuestados del segundo grado de educación secundaria de la IE Mariscal Castilla, el 59\% se encuentra en un nivel inicial, el $32 \%$ se encuentran en proceso y se evidencia que el $9 \%$ está en el nivel satisfactorio con respecto a las características del texto argumentativo.

Frente a estos resultados se planteó la propuesta de una estrategia motivacional para mejorar la producción de textos argumentativos. 


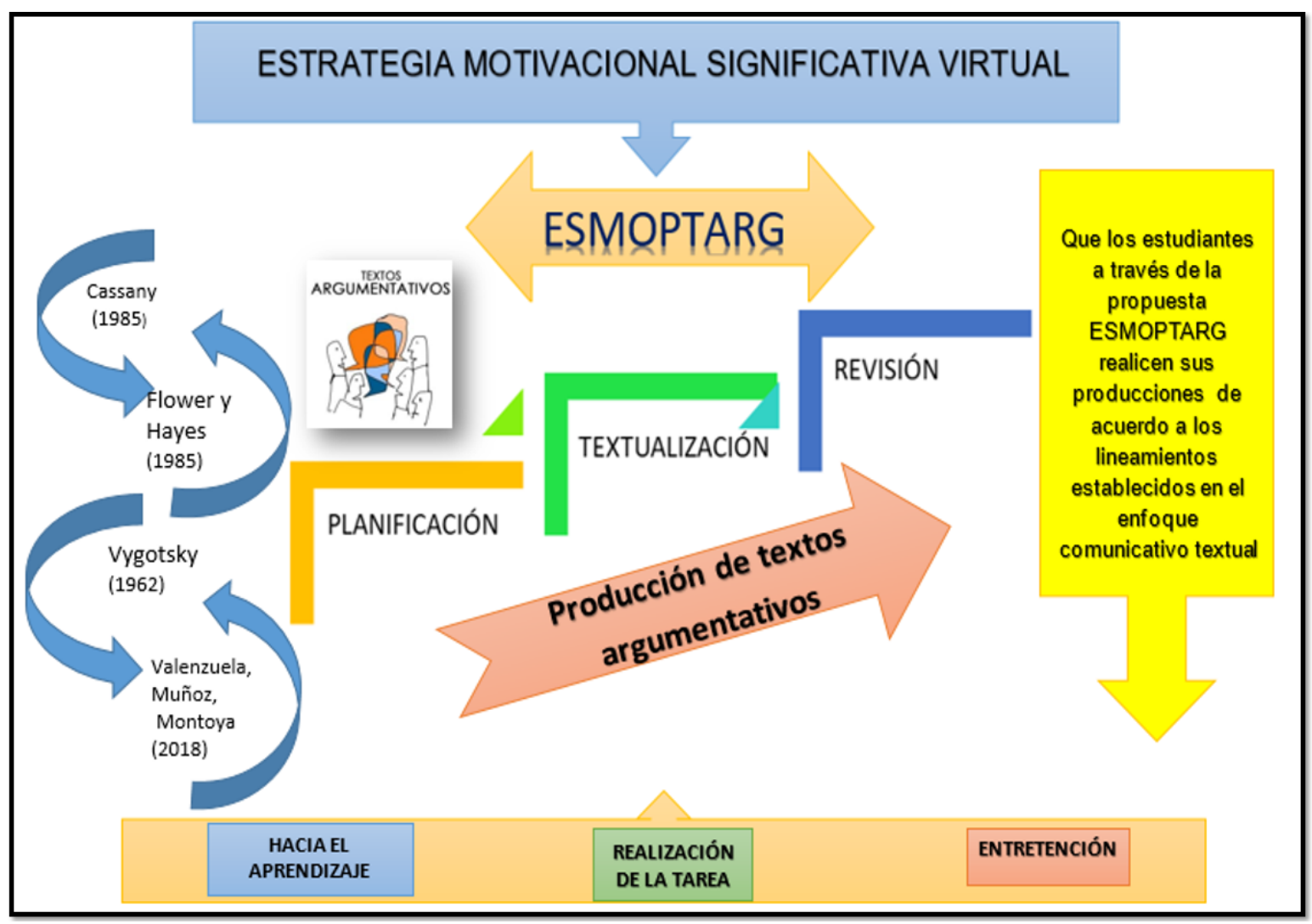

El presente programa está dirigido a aplicar estrategias motivacionales para promover la producción de textos escritos siguiendo los procesos de planificación, textualización y revisión, para ello se desarrollarán diez actividades de aprendizaje que se detallan.

\section{PRODUCCIÓN DE TEXTOS ARGUMENTATIVOS}

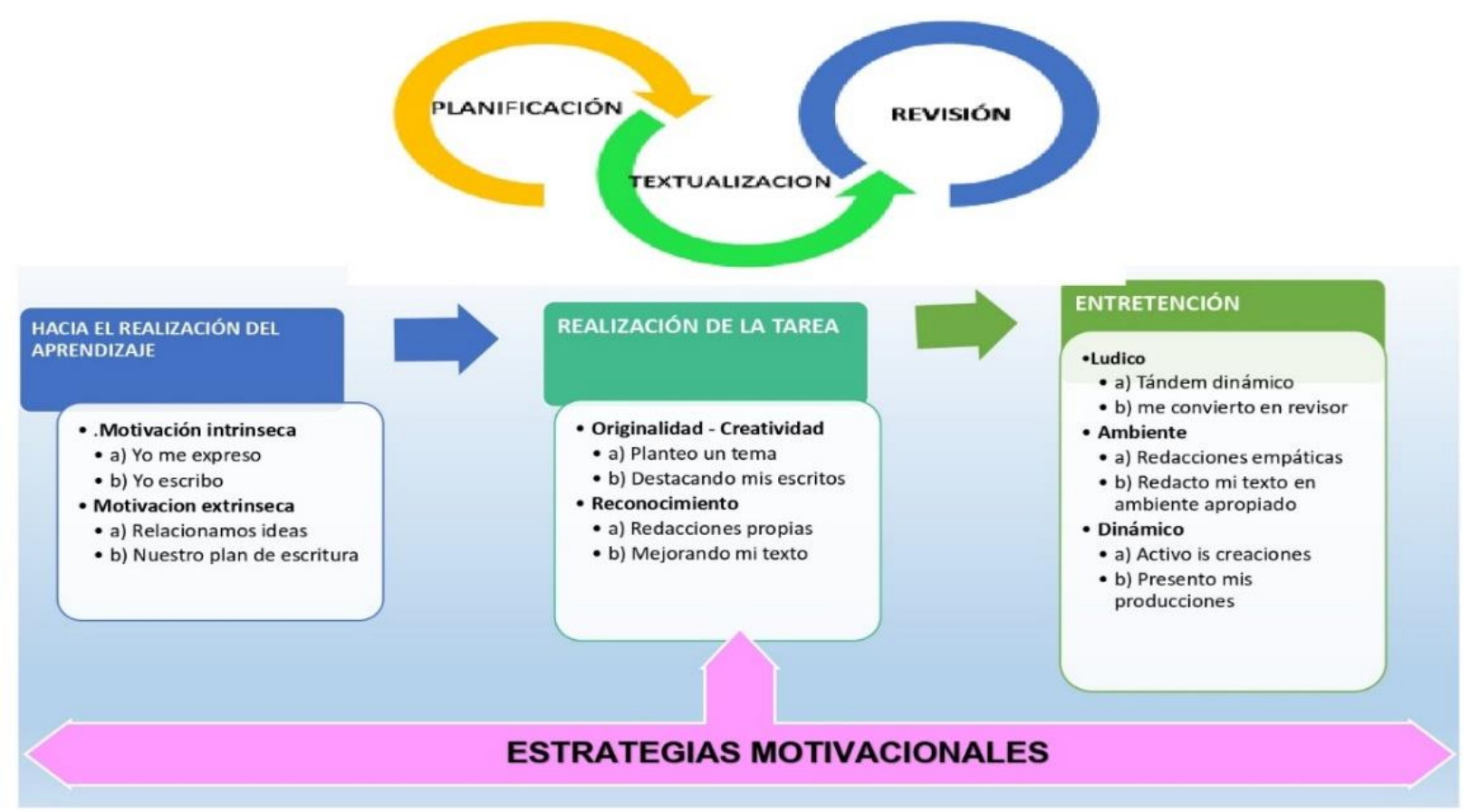


El impacto de la propuesta es fortalecer los niveles de aprendizaje de los estudiantes. La ECE 2019, ubica a la UGEL Paita en la cuarta posición a nivel de región Piura respecto a la comprensión lectora con $47,7 \%$ en inicio, 27,1\% en proceso y $11,2 \%$ en satisfactorio. Los estudiantes con competencias escritas, desarrollarán la investigación y difundirán sus publicaciones por escrito, además esta propuesta, permite desarrollar en los estudiantes la capacidad de reafirmar sus ideas con juicios propios, así como conocer diversas situaciones comunicativas a nivel oral y escrito.

De esta manera, sus aportes cognitivos serán muy importantes para la comunidad educativa y del contexto en que se desenvuelve; es necesario acentuar la importancia del lenguaje escrito como factor primordial del pleno potencial humano y del dominio de los códigos de la sociedad moderna y sus cambios.

Esta idea es fortalecida por Pinzás (2010) cuando manifiesta que las instituciones escolares estarán puestas en la convergencia entre aprendizaje del manejo del lenguaje escrito y desarrollo de habilidades cognitivas y afectivas, es decir un estudiante debe aprender a leer y comprender lo leído, así como redactar sus textos con cohesión y coherencia desarrollando estructuras cognitivas y afectivas que lo comprometen como un ser que se enfrenta y resuelve los problemas que se presenten.

\section{Discusión}

La investigación, referida a realizar una propuesta de estrategia motivacional significativa en la producción de textos argumentativos en estudiantes de segundo grado de secundaria de una institución educativa de Paita, según los resultados, se puede evidenciar que más de la mitad de los estudiantes (59\%), se ubicaron en un nivel de inicio en el desarrollo de la producción de textos argumentativos, lo que significa que los estudiantes tienen dificultad de evidenciar aprendizajes esperados en redacción y solo logran realizar textos poco exigentes de lo que se espera para el grado, se comprueba dificultades en el desarrollo de la tarea por lo que necesita mayor acompañamiento e intervención del docente.

Otro grupo importante (32\%) se ubicó en un nivel de proceso, lo que demuestra que los estudiantes están próximos o cerca del nivel esperado, pero aún requiere acompañamiento; se puede observar que los estudiantes evaluados presentan problemas en la planificación, producción y revisión de escritos, copian ideas de manera literal, tienen dificultad para integrar las opiniones en forma adecuada, presentan vacíos en definir claramente una tesis, redactan argumentos poco consistentes al defender sus ideas.

Los hallazgos concuerdan con los encontrados por Culquicondor (2017), quien en su investigación concluye que los estudiantes que terminan la educación básica tienen un bajo nivel de logro (68.8\%) al momento de producir textos argumentativos a nivel de superestructura, macroestructura y microestructura situación que estaría asociada a su nivel de práctica lectora, iguales resultados encontró Medina (2019), ya que concluye que antes de aplicar la estrategia de lectura la mayor parte de los estudiantes $85 \%$ tienen dificultades serias al momento de producir textos haciendo uso de la argumentación.

En cuanto a la dimensión propiedades del texto argumentativo, más de la mitad de los estudiantes (76\%) se ubicó en un nivel de inicio, lo que significa que los estudiantes presentan dificultades para el desarrollo de la propiedad de adecuación, mostrando problemas al escribir para persuadir y convencer al lector, se percibe deficiencia en fundamentar su punto de vista.

En la propiedad coherencia textual la mayor parte de los estudiantes tienen dificultades para seleccionar ideas relevantes, no considera la tesis y tampoco propone argumentos, lo mismo sucede con la propiedad cohesión textual, los estudiantes muestran deficiencias para organizar su discurso, poco uso de conectores aditivos, de tiempo y lugar, de conclusión y de conectores comparativos en los argumentos; esto nos permite señalar que este grupo de estudiantes que se ubicó en el nivel inicio necesita mayor tiempo de acompañamiento e intervención del docente teniendo en cuenta su ritmo y estilo de aprendizaje.

Los resultados concuerdan con lo encontrado por Zambrano, Orozco y Caro (2017), ya que encontraron problemas serios en la redacción de textos argumentativos, no habiendo una coherencia textual adecuada, errores en la utilización de conectores textuales tanto de inicio como de procesos y muestran una débil postura al defender en el desarrollo de su texto.

Respecto a las teorías descritas lo encontrado en la investigación discrepa con lo que plantea Romero (2009), Casado (2002) y Cassany, Luna y Sanz (2007), los autores señalan que la coherencia textual, es la conexión de las partes en un todo e implica la unidad necesaria para la inteligibilidad y aceptabilidad del texto, aspectos que no se cumplen en el grupo estudiado ya que la mayoría de los investigados se ubicaron en la categoría de inicio. 
Los resultados referidos a la segunda dimensión procesos de escritura; la mayor parte de los estudiantes (65\%) se ubicaron en la evaluación realizada en un nivel de inicio, lo que se evidencia en el indicador planificación del texto, mostrando dificultades en la aplicación de reglas gramaticales, la mayor parte de los estudiantes cometen errores en lo que respecta al uso de reglas de ortografía literal, puntual y acentual, hay dificultades, en cuanto al indicador revisa el texto, presentan deficiencias en textualización, elaboran un solo borrador de sus producciones y como no los usan cometen errores textuales, y en la revisión final de su producción, no suelen tener la costumbre de revisar cuidadosamente lo que están escribiendo.

Hallazgos similares se encontraron en la investigación propuesta por Demarini (2017), quien concluye que, al inicio de la investigación, los estudiantes presentaron nivel bajo en el factor coherencia textual (10.38, media), en el factor planificación textual, los datos determinan un nivel medio con una media de 11.50 y en la cohesión textual igualmente se evidencia dificultades con una media de 10.30 .

Portal (2010) Figueroa y Simón (2011) concuerdan en que la mayoría de estudiantes al producir un texto deben desarrollar el proceso de planificación, cumplir con la programación de actividades para la elaboración del texto, esta capacidad permite estar anticipados en la forma, el contenido, el tema, el tipo de texto que se pretende desarrollar, los recursos textuales que se van a utilizar y hasta los argumentos que tendrá el texto; sin embargo en el presente estudio no se pudo encontrar un buen desarrollo de esta dimensión, siendo necesario la utilización de estrategias motivacionales para la producción de textos argumentativos.

Portal (2010) y MINEDU (2017), hacen énfasis que para lograr los procesos de escritura para una buena producción de textos argumentativo es necesario movilizar un conjunto de saberes, conocer modelos textuales, saber organizar ideas, manejar el vocabulario adecuado, llevar una secuencia lógica, relacionar enunciados de manera coherente, escribir con corrección ortográfica (puntuación y tildación).

Finalmente, la dimensión característica del texto, según los resultados encontrados se pudo evidenciar que la mayor parte de los estudiantes se ubicó en un nivel de inicio, $59 \%$ de los estudiantes evaluados, lo que permite vislumbrar que hay dificultades en los logros previstos para esta dimensión, es difícil encontrar un título adecuado para sus producciones, deficiencias en desarrollar argumentos en la introducción, en el cuerpo del trabajo casi no usan citas de referencia para sostener sus argumentos, en cuanto al indicador conclusiones.

Siempre presentan dificultades para elaborarlas y no guardan relación con el contenido del documento redactado; estos resultados ponen en evidencia la necesidad de un acompañamiento por parte del docente, además de ver los ritmos y estilos de aprendizaje del grupo estudiado.

Estas dificultades podrían estar asociadas al contexto donde se desenvuelve el estudiante no siendo este tan favorable, presentando dificultades en su competencia argumentativa; según la teoría Sociocultural de Lev Semionovich Vigotsky; reconoce que el aprendizaje es un proceso personal, pero afirma que el autoconstrucción de conocimientos se logra en la interacción entre el sujeto y el contexto sociocultural en que se desenvuelve, el que luego se internaliza.

Resultados muy similares encontró Torres (2016), la investigación logró determinar el nivel de la competencia argumentativa fue de inicio, el $64 \%$ de ellos obtuvieron notas entre 0 a 10) después de la aplicación este resultado mejoró por lo que se demostró el efecto de la aplicación del Programa en el desarrollo de la Competencia Argumentativa de las estudiantes del 4to. grado.

\section{CONCLUSIÓN}

Al analizar los resultados de producción de textos argumentativos, aplicada a los estudiantes de segundo grado de educación secundaria, que fueron bajos en relación al grado que cursan, es que se concluyó en la importancia de desarrollar un programa de estrategias motivacionales para mejorar la producción de textos argumentativos.

Gracias a la aplicación del instrumento de evaluación se confirmó que, el nivel de producción de textos argumentativos, no es el adecuado para los estudiantes de segundo grado de secundaria, ya que se encontraron resultados en un niel de inicio, tanto en las propiedades del texto, procesos de escritura y características del texto argumentativo.

Es por esta razón que se reafirma la elaboración de un programa de mejora de producción de textos argumentativos, utilizando estrategias motivacionales, para los estudiantes de segundo grado de secundaria; ante los bajos niveles de producción de textos argumentativos tanto en las propiedades del texto argumentativo, procesos de escritura y propiedades del texto argumentativo. 


\section{BIBLIOGRAFÍA}

1. Alcázar Barragán, $C$ y Atencia Molina, M. (2020-11-11.). Escribo con argumentos: secuencia didáctica como aporte al mejoramiento de la escritura de textos argumentativos en estudiantes de grado décimo de la Institución Educativa la Ribera de la ciudad de Montería. Facultad de Educación y Ciencias Humanas.

2. Baño Caiza, Lida María (2020). La enseñanza de la lengua española en la producción de textos escritos [tesis] Universidad Técnica de Ambato. Ecuador. Disponible en: https://repositorio.uta.edu.ec/bitstream/123456789/31652/1/0503055881\%20Lida\%20Mar\%c3\%ada\%20Ba\% c3\%b10\%20Caiza.pdf

3. Bolívar Romero, A. (2014). Producción de textos argumentativos escritos en estudiantes de décimo grado. Escenarios, 10(2), 92. https://doi.org/10.15665/esc.v10i2.223

4. Bos, M. S., Viteri, A., \& Zoído, P. (2019). Pisa 2018 En América Latina ¿Cómo nos fue en lectutar? BID, CIMA. Pisa 2018., I, 1-4. https://doi.org/10.31244/9783830991007

5. Camps, A. (1995). Aprender a escribir textos argumentativos: características dialógicas de la argumentación escrita. Comunicación, Lenguaje y Educación, 7(2), 51-63. https://doi.org/10.1174/021470395321340439

6. Cárdenas P., A. (2017). Lingüística del lenguaje: hacia una pedagogía de la escritura. Revista Folios, 25, 70. https://doi.org/10.17227/01234870.25folios 70.80

7. De La Torre Holguín, J. G. (2019). El nuevo enfoque comunicativo textual del curso de Lenguaje. Revista Helios, 3(2), 269-278. https://doi.org/doi.org/10.22497/Helios.32.3205

8. Figueroa., M. R., \& Simón., P. J. (2011). Planificar, escribir y revisar, una metodología para la composición escrita. Una experiencia con estudiantes del Instituto Pedagógico de Caracas (IPC) Planning, writing and revising : a methodology for written composition. An experience with studen. Revista de Investigación Instituto Pedagógico de Caracas, 35(73), 119-147.

9. Flower, L., \& Hayes, J. R. (1980). The Cognition of Discovery: Defining a Rhetorical Problem. College Composition and Communication, 31(1), 21. https://doi.org/10.2307/356630

10. Gutiérrez Pérez, Blanca N y Uceda Duclos, Santiago Alberto (2017). Modelo didáctico basado en el aprendizaje significativo y el desarrollo de capacidades comunicativas en estudiantes universitarios. Revista Ciencia y Tecnología, v.13, $\quad$ n. $33-44, \quad 2017.2$ Disponible en: https://revistas.unitru.edu.pe/index.php/PGM/article/view/1872/1800

11. Guzmán-Cedillo, Y. I., Flores-Macías, R. del C., \& Tirado-Segura, F. (2013). Desarrollo de la competencia argumentativa en foros de discusión en línea: Una propuesta constructivista. Anales de Psicologia, 29(3), 907916. https://doi.org/10.6018/analesps.29.3.175681

12. Hymes, D. (1972). On Communicative Competence In: J.B. Pride and J. Holmes. (Eds) Sociolinguistics. Selected Readings, 269-293.

13. Merma Herrera, M. L. (2018). Programa de producción de textos narrativos, para contribuir al desarrollo de la creatividad textual en estudiantes del iv ciclo de educación primaria de la institución educativa multigrado $\mathrm{N}^{\circ}$ 40433 del distrito de Yanaquihua-Arequipa 2018.

14. MINEDU. (2020). Inicio | SIAGIE. MINEDU.

15. Morales, L. J. G., \& Ramírez, D. J. S. La producción colaborativa de textos argumentativos en el desarrollo del pensamiento crítico en estudiantes de grado once de la institución educativa Francisco José de Caldas, Santa Rosa de Cabal, Risaralda.

16. Pérez F., M., Quijano R., R., \& García M., I. (2019). Desarrollo de la competencia comunicativa en estudiantes de bachillerato. 7(January), 1-10. http://journals.epistemopolis.org/index.php/ educación

17. Román F., I., \& Zapata F., M. (2019). Analysis of argumentative texts prepared by chilean students of pedagogy. Educacao e Pesquisa, 45, 1-19. https://doi.org/10.1590/S1678-4634201945197601

18. Rosado R., R. (2012). Re $\quad$ Textos Ihttp://www.prepaaguascalientes.sep.gob.mx/prepaabierta/archivos/modulo_13.pdf,teratura/borrador/pdf/argu mentacion.pdf

19. Villasmil, Y., Arrieta, B., \& Fuenmayor, G. (2009). Análisis de la comprensión lectora y producción escrita de los estudiantes de educación media diversificada y profesional. Multiciencias, 9(1), 62-69. http://www.redalyc.org/articulo.oa?id=90411683009

20. Weston, A. (2005). Las Claves De La Argumentacion (Ariel) (Spanish Edition). 